\title{
A paper-format group performance test for measuring the implicit association of target concepts
}

\author{
KAZUO MORI \\ Tokyo University of Agriculture and Technology, Tokyo, Japan \\ AKITOSHI UCHIDA \\ Shinonoi-nishi Junior High School, Nagano, Japan \\ AND \\ RIKA IMADA \\ Shinshu University, Nagano, Japan
}

\begin{abstract}
We developed a paper test utilizing a mechanism for measuring implicit association similar to that used in the Implicit Association Test (IAT; Greenwald, McGhee, \& Schwartz, 1998). The target concepts were buried among positive and negative words on a piece of paper. Examinees marked the targets as "bad" or "good" in one task and conversely in the other, along with the evaluative words. Instead of reaction times, we counted the number of words marked in $20 \mathrm{sec}$ for each task. This procedure allowed group administration. We calculated the implicit measure using the difference in the average number of words marked in the task pairs. The results of a test administered to 82 undergraduates with three different targets showed significant correlations $(r \mathrm{~s}=.26-.35)$ with the results of IAT administered to the same participants. It also showed significant reliability $(r \mathrm{~s}=.56-.71)$. We discuss the practical usability of the test with application studies conducted in various areas.
\end{abstract}

Several new methods have been developed to assess attitudes or beliefs of persons by measuring their implicit associative processes, which are assumed to be beyond their conscious control (Fazio, Sanbonmatsu, Powell, \& Kardes, 1986; Greenwald, McGhee, \& Schwartz, 1998; Williams, Watts, MacLeod, \& Mathews, 1988). Among them, the most influential one has been the Implicit Association Test (IAT) developed by Greenwald and his collaborators (Greenwald et al., 1998).

Nosek, Greenwald, and Banaji (2007) recently reviewed the test 7 years after its original publication, writing that "the IAT has been applied in a diverse array of disciplines including social and cognitive psychology, clinical psychology, developmental psychology, neuroscience, market research, and health psychology" (p. 267; references in each discipline are omitted here).

However, the IAT's usage is still limited because it is only administered individually, using a computer. Some researchers have developed paper-format versions of the IAT (e.g., Lane, Mitchell, \& Banaji, 2005; Lemm, Sattler, Khan, Mitchell, \& Dahl, 2002; Lowery, Hardin, \& Sinclair, 2001; Schwartz, O'Neal Chambliss, Brownell, Blair, \& Billington, 2003; Teachman, Gapinski, Brownell, Rawlins, \& Jeyarams, 2003). Lowery et al. argued that the crucial merit of the IAT, the fact that it is not susceptible to participants' intentional processing, is maintained in the paper-format IAT. Lemm et al. tested the reliability and validity of two versions of paper-format IATs, with picture or verbal stimuli, by comparing their results with those of computer-based IAT. They concluded that a paper-format IAT measuring attitudes on race yielded similar results to those of a computer-based IAT when both measures used verbal stimuli.

Nevertheless, the application of the paper-format IAT has been limited to just a few studies, in comparison with the numerous application studies of the original computerbased IAT. This is mostly because the ease of use of the paper-format IATs does not offset their relative weakness in precision. The paper-format IAT was a simple conversion of the original IAT into a paper format. The categorization tasks of the original IAT were done on lists of words printed on separate sheets. Therefore, the test needed to be formatted as a booklet of 10 to 12 pages. Even with these repeated task performances, the critical measurement was done only once. Because itemwise measurement was not possible in the paper-format IAT, only the overall speed was assessed for each categorization task. In principle, it might be possible to repeat the measurements, but it would require test booklets with double or triple the number of pages.

K. Mori, kaz-mori@cc.tuat.ac.jp 
The present article describes a newly developed procedure named the Filtering Unconscious Matching of Implicit Emotions ("FUMIE") Test, with which we were able to overcome the limits of the computer-based IAT while converting to a simple paper format. The FUMIE Test is basically a paper-and-pencil version of the IAT on a single sheet of paper. The preparation of test sheets is labor saving, requiring neither sorting pages nor stapling. The test measures performance speed rather than response time repeatedly. Therefore, it can collect more reliable data and can be administered to a group of examinees easily. In the FUMIE Test, the target concept being measured is presented directly in the form of category words rather than category members. The test consists of two categorization tasks of a single attribute (e.g., evaluation). In one task (the positive task), participants are instructed to categorize the target as good, along with other positive words, and negative words as bad, and to do the reverse for the other task (the negative task). The categorization speed is assessed by number of items completed in $20 \mathrm{sec}$ for each task. The positive and negative tasks are repeated five times, to obtain more stable average scores. The implicit measure of the participant's attitude toward the target concept is calculated by comparing the difference in the average number of items completed on the two tasks in a manner similar to that accomplished by the IAT.

In this article, we first report precisely the administrative procedures of the FUMIE Test. Then, we present the results of validity and reliability assessments of the test. We also discuss the practical usability of the test by reviewing some application studies in which the test has been utilized.

\section{DESCRIPTION OF THE FUMIE TEST A Group Performance Paper-and-Pencil IAT}

\section{Construction of the FUMIE Test}

FUMIE Test sheet. The Japanese version of the FUMIE Test consists of 60 words, of two Chinese characters each, arranged in 13 rows on an A3-sized piece of paper $(29.70 \times$ $41.99 \mathrm{~cm}$ ) (see Figure 1). Because the Japanese words all consist of two Chinese characters, the length of the rows, as well as their appearance, is unchanged regardless of the variety of words used in the rows. Because of this specific trait of the Japanese language, the Japanese versions of the FUMIE Test are made up of 13 rows of words of almost identical appearance. Since word length varies in English, it is recommended that rows and columns in an English version are used so that the words are arranged in columns rather than in rows, as in the paper-format IAT (Lane et al., 2005). The examinees will mark either a circle or a cross on each word, going downward rather than from left to right. (For making an English version, replace "rows" with "columns" in the following sections.)

Evaluative words used in the FUMIE Test. The 30 pairs of words were selected to meet three criteria: (1) consisting of two Chinese characters, (2) forming an antonym pair, and (3) being familiar to even junior high school students. Then, forty 7 th-grade (junior high school) students, 21 males and 19 females, rated the 30 pairs of words as to whether they were positive or negative. Sixteen pairs were finally selected because they were familiar to all the students and were classified as expected by all of them. The 16 pairs and their equivalent English words are shown in Appendix A. (For an English version, the evaluation words should be selected and tested for familiarity using the same procedure as was described here.)

Arrangement of evaluative words and the target. In the first row of the FUMIE Test, each of these 16 pairs of words appears randomly once or twice. More precisely, the words were placed so that three positive and three negative words should appear randomly in every six sets of words. This first row is to be used for a classification practice task.

The following 12 rows (2nd-13th) are to be used for the implicit association measurement. The target word for which the implicit association value is to be measured is interspersed 20 times among the positive and negative words in each row. Two pairs of rows (2nd-3rd and 4th5 th) are made with the same principle but with words and target placed randomly. In each row of 60 words, 20 are the targets, 20 are positive words, and 20 are negative words, arranged in the same manner as the first row, except the target word is inserted so that every combination of 3 words is composed of "the target, one positive word, and one negative word," in a random order without any immediate repeat of the targets. The 2 nd and 4 th rows are used for the positive tasks, in which examinees mark a circle $(O)$ on the target, and the 3rd and 5th are used for the negative tasks, with a cross $(X)$ to be marked on the target. (Marking a circle or a cross is the most commonly used procedure for referring to "good" and "bad" in Japanese culture. Even preschoolers are familiar with this marking custom.)

Then, the 2 nd and 3 rd rows are duplicated as the 7 th and 6 th, and the 4 th and 5 th rows as the 9 th and 8 th. In this way, the rows used for the positive tasks (the 2nd and 4th) are also used for the negative tasks (the 7th and 9th), and vice versa. The four rows from the 2 nd to the 5 th are further duplicated for the 10th to the 13th, in the same order.

Easy replacement of the target for a new measurement. The preparation of the test sheet may seem complicated. However, once the prototype file is made, using an Excel format, the test sheet for a new target can be made easily by replacing the target words using the replacement function. We recommend printing the test sheet in A-4 size and then enlarging it to A-3 size using either a copy machine or an office or commercial printer at the time of mass printing.

\section{Administration of the FUMIE Test \\ Administration time and apparatus. The FUMIE} Test can be administered to a group, such as a school class or group of attendees at a meeting or seminar. It takes about $7 \mathrm{~min}$ to administer the test, including a general instruction session. It is also possible to use it in conjunction with an explicit measure such as a questionnaire, or with a scholastic written examination. Using a stopwatch is recommended for measuring the $20 \mathrm{sec}$, although a wristwatch with a second hand can also be used. 


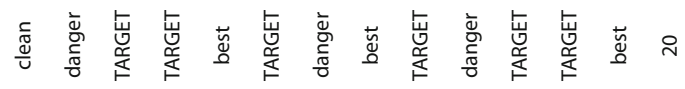

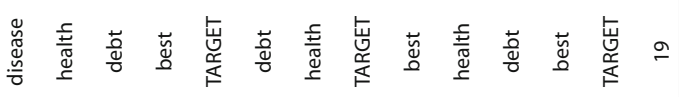

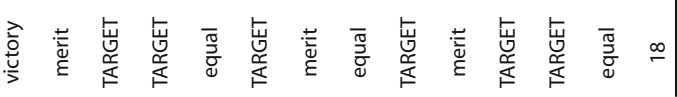

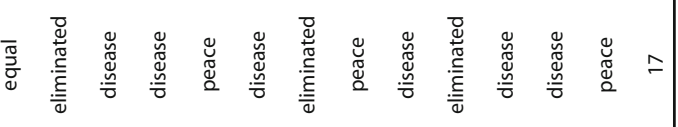

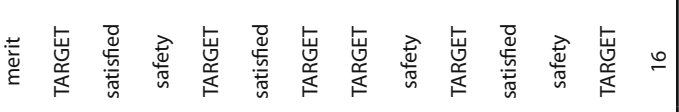

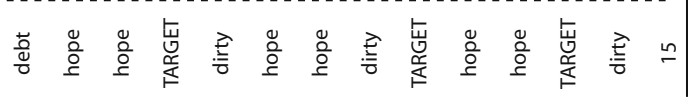

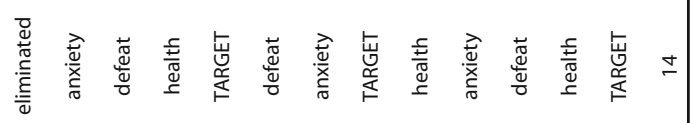

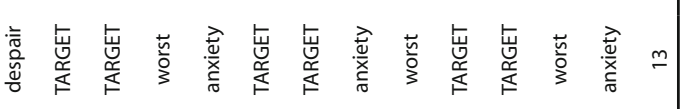

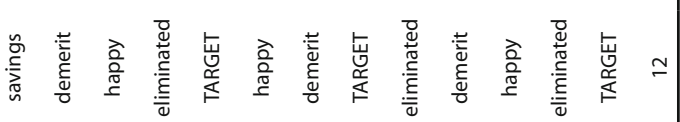

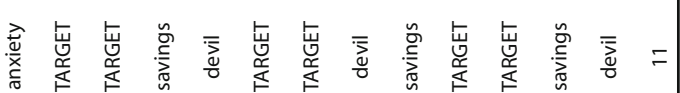

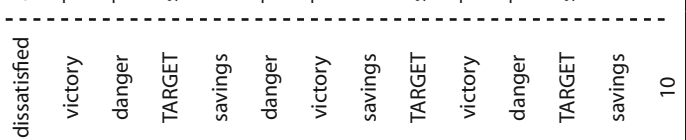

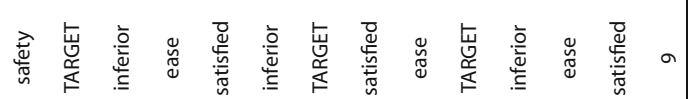

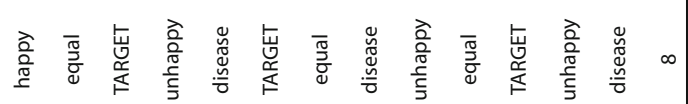

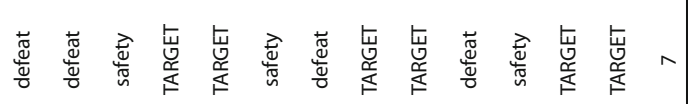

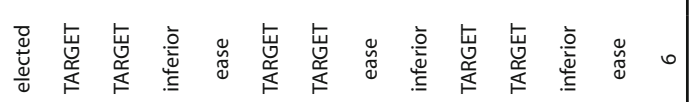

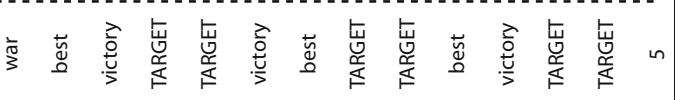

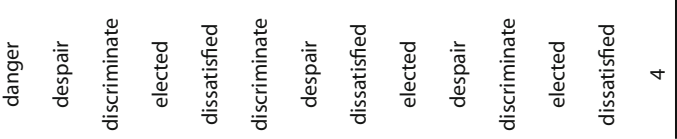

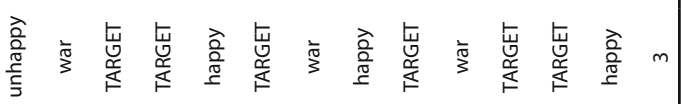

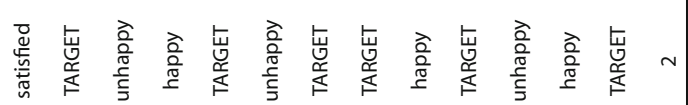

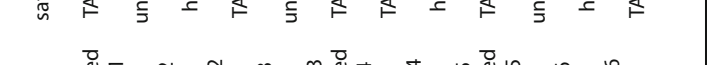

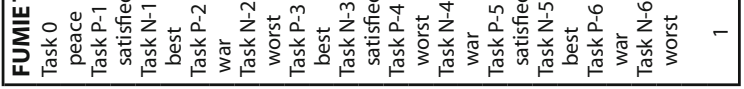

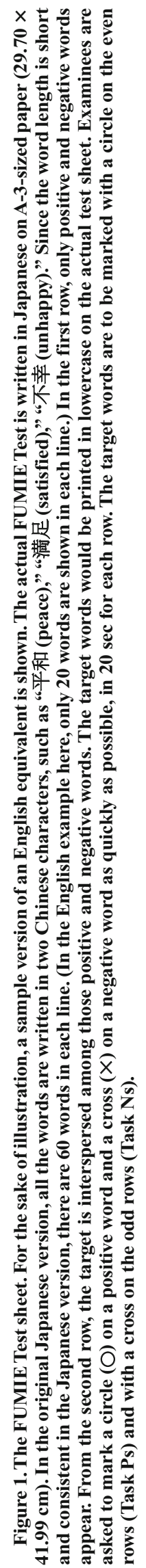


Informed-consent procedures. For ethical reasons, all the examinees of the FUMIE Test should be fully informed of the possible disclosure of their implicit attitudes before the administration of the test. The administrator must inform them that the tests may reveal their implicit attitudes and that they can stop participating any time they want to do so.

General instructions. Before handing out the test sheet to examinees, instruct them to clear the table so that an A-3sized sheet of paper can be placed on it with no obstructions for the speed performance test.

The following general instructions are given at the beginning.

Words with positive meanings, such as "success" and "victory," and those with negative meanings, such as "failure" and "defeat," are arranged in lines from left to right. Your task is to mark a circle $(\bigcirc)$ on the positive words and a cross $(X)$ on the negative words, as quickly as possible, with as few errors as possible. If you notice an error, just re-mark correctly nearby. Do not use an eraser. At the "Start" signal, proceed from left to right without skipping any words. At the "Stop" signal, stop the task and wait for the next instruction.

Measuring procedures. After the general instructions, the first task will start. This is a simple classification task of positive and negative words. The first task serves as a practice. The administrator should make sure all the participants are ready and give the start signal as clearly as possible. After measuring $20 \mathrm{sec}$, the administrator should announce the stop signal.

Before the second task, the following additional instruction is given.

In the next task, there is a "target" word among the positive and negative words you just classified above. As for the "target," you should mark a circle $(\bigcirc)$ on it along with the other positive words. All the other instructions are the same as for the first task.

Again, after checking whether all the participants are ready, the administrator will give the start and stop signals with a 20 -sec interval.

The instruction for the third task is as follows.

In the next task, you should switch the mark of the "target" and put a cross $(\times)$ on it along with the negative words. Mark a cross on the "target" rather than a circle. All the other instructions are the same as for the previous tasks.

These two tasks serve as positive and negative tasks, respectively, which are similar to the congruent and incongruent tasks of the IAT. For the 4th to 11th tasks, the same procedure is repeated. In this way, five pairs of positive and negative tasks are administered. After finishing the 11th task, the administrator will declare the end of the test and collect the test sheets.

There will remain two rows for the 12th and 13th tasks. These are dummy tasks, to prevent the examinees from detecting the end of the series of tasks so they will not make an extra effort on the last task.

\section{Calculation of Implicit Association Scores}

Implicit association scores. The implicit association score (IAS) is calculated with the following equation: IAS $=$ (average number of items completed on positive tasks) - (average number of items completed on negative tasks).

The IAS is the basic measure of the FUMIE Test. The IAS becomes a positive value when the target is implicitly associated with positive words rather than negative words, and a negative value if it is associated in the reverse way. An equivalent measure of the IAT, such as "D scores" (Greenwald, Nosek, \& Banaji, 2003), can be calculated with mean latency per item by dividing the phase duration $(20,000 \mathrm{msec})$ by the average number of items completed on both tasks. However, the IAS is preferable to those sophisticated measures in the sense that the FUMIE Test can be used on a variety of occasions and for various purposes for data collection without using a computer. The simple difference between the two focal tasks is also easier to understand than other measures, such as ratio, or reaction times in milliseconds, for schoolteachers and other laypersons who are not familiar with statistical terms.

Calculation of average number of items completed. Although there are five pairs of positive and negative tasks on each completed test sheet, only the last four pairs are used in the calculation of the averages. The performances of the first pair of tasks, especially the very first of all the tasks, may contain effects of the initial effort and/or bewilderment of examinees. Therefore, it should be safe to use the scores obtained in the middle. It should be noted that the possible effect of a testee's special effort at the end is also eliminated by unpredictable ending of the test after the 11th row, as stated above. As reported in the actual studies below, undergraduates generally perform 25 to 35 items per task (in $20 \mathrm{sec}$ ), on average, with the $S D$ s around 6 (see Appendix C).

Duration problems of marking $\bigcirc$ and $X$. The responses required in the FUMIE Test, marking a circle or a cross on each word, have a measurable duration even if this is done as quickly as possible, compared with the simple key touching in the IAT procedure. Because of this duration in responding, there may be an incomplete marking at the time of the stop signal, such as a fraction of a circle or a single slash instead of a complete $\times$. These incomplete marks are regarded as valid responses and counted.

The response duration in drawing has a more fundamental problem. A cross is drawn in two strokes, whereas a circle is drawn in one stroke (though it is longer). It might be quicker to mark a circle than a cross. If so, more words marked in the positive tasks could be attributed partly to the quicker drawing of circles.

Two supplementary studies revealed that, although the drawing time for a cross seems slightly longer than that for a circle, the time difference should not cause any substantial difference in the test performance. (See Appendix B for the details of the supplementary studies.)

Treatment of errors. It is worth noting here that the error check must be done by a human in the FUMIE Test. The evaluation of the cost-performance trade-offs of the treatment of errors suggests that the most suitable proce- 
dure is to simply count the number of items completed, regardless of errors. Fortunately, with the administration of FUMIE Tests to various age groups of examinees-from junior high school students to senior citizens - it has been found repeatedly that the error rates are negligibly low. The average rates of error responses were less than $1 \%$ among undergraduate participants for monopolar versions. As for cognitively more demanding bipolar versions, the error rates were around 2\% among undergraduates and about 3\%-4\% among the senior citizens (see Appendix C; the mono- and bipolar versions will be explained shortly). It has also been proven that the elimination of error responses did not show any practical difference in the interpretation of test results. Therefore, it would be wise to simply count the number of items completed, without checking their correctness.

We recommend, however, surveying the overall error rates of the group by checking the records of $5 \%$ to $10 \%$ of the random samples of the examinees. If the error rate is higher than 5\% among the samples, the credibility of the test data overall comes into question. The examinees may not have taken the test seriously.

Treatment of omissions and other abnormalities. Incidental omissions, rather than errors, should simply be discounted from the scores. However, intentional omissions should invalidate the test as a whole. Intentional omissions include a successive omission of items, omissions of a particular word or the target, neglecting all the positive or negative words, and so on. If there is an intentional omission in even one of the tasks performed, it would be better to discard the whole record of the examinee.

There may be an incidental skipping of a whole line. The examinee may have skipped the line by mistake. In this case, one extra line should be performed on the 12th, which would have been left blank otherwise. It can be treated as a simple misplacement of the task line, if that is the case. A test paper with omissions of whole lines, without a sign of simple replacement, should be treated as invalid.

There may be other abnormalities, such as excessive performances (marking all 60 words within $20 \mathrm{sec}$ ), extremely low performances (less than 10 items), too much fluctuation among the tasks (e.g., 15 items on one task and 50 on another), or any other unnatural performance patterns that show artificiality (e.g., the same number of items marked on all the tasks). To date, for such aberrations, no criteria can be set theoretically, nor any based on sufficient empirical data. It is recommended that examiners discard such dubious test papers for the sake of the overall reliability of the data.

\section{Monopolar and Bipolar Versions of the FUMIE Test}

The FUMIE Test described above is a monopolar version, which can measure the implicit association of any single target concept. The monopolar version of the FUMIE Test has evolved from a bipolar version, in which the targets were paired, such as "black vs. white" or "male vs. female." A bipolar version of the FUMIE Test can be made by inserting the two target words randomly among the evaluative words. The instructions to the examinees for the test tasks must be changed accordingly.
Bipolar versions are suitable for those researchers who want to compare opposite-concept pairs in a single test administration. We would recommend that the "presumed positive target" be marked with a cross first. This would be equivalent to the incongruent-congruent order of the IAT administration, in which larger IAT effects would be expected than in the reverse order. However, the procedural order would not be crucial in the FUMIE Test administration. The effects of the task order can be examined at the time of the analyses. Since two tasks are repeated five times each, interchangeably, diagrammed as "PNPNPNPNPN," four pairs of both the PN and NP orders can be obtained by discarding either the first two or the first and last ones, yielding either "[PN]PNPNPNPN" or "[P]NPNPNPNP[N]," respectively.

It should be noted that the bipolar tests can reveal only the relative difference in the implicit associative values between the targets. Mori (2003) reported that a bipolar FUMIE Test on blacks versus whites, administered to Japanese participants (47 junior high school students, 49 undergraduates, and 52 adults), showed similar associative patterns as were found on the IAT administered to white American participants. However, the results should not be interpreted to mean that the Japanese people have negative implicit association against blacks. As Mori, Imada, and Fukunaka (2005) reported, two separate monopolar FUMIE Tests, administered to different Japanese participants (33 high school students and 34 undergraduates), revealed that the average IASs for blacks and whites were both positive but had different magnitudes, $0.2-1.4$ and 2.4-2.8, respectively. The strong positive attitude toward whites could have concealed the weak positive attitude toward blacks if the bipolar versions had been administered to the Japanese participants.

\section{VALIDITY AND RELIABILITY OF THE FUMIE TEST Correlation With the IAT}

\section{Method}

Participants. Eighty-two undergraduates (32 males and 50 females, 20-23 years old) took both the FUMIE Test and the IAT. All of them were native Japanese. They participated in the experiment voluntarily and were informed that the tests would reveal their implicit attitudes and that they could stop participating any time they wanted to do so.

FUMIE Test. Three different versions of FUMIE Test sheets were prepared using three target words: “恋愛 (romantic love),” “結婚 (marriage),” and “妊娠 (pregnancy).” These targets were selected because they should evoke strong affective feelings, both implicitly and explicitly, among young students.

The participants took the three FUMIE Tests during three regular psychology classes, with one target per class, in the order "marriage," "pregnancy," and "romantic love."

Modified IAT. A standard IAT program distributed by Millisecond Software was partly modified so that the results could be directly compared with those of the FUMIE Test. The modified IAT consisted of only three blocks: 
(1) concepts-only task, (2) congruent task, and (3) incongruent task. In the concepts-only task, 16 pleasant and 16 unpleasant words appeared randomly only once. With the congruent and incongruent tasks, the target words appeared eight times each, randomly interspersed among the 32 pleasant and unpleasant words. In the congruent task phase, participants were instructed to respond to the target and the pleasant words with the same key, whereas they were instructed to respond to the target and the unpleasant words with the same key in the incongruent task. The order of the congruent and incongruent tasks was counterbalanced between participants. The procedure was almost equivalent to the single-category IAT introduced by Karpinski and Steinman (2006), except that only a single target word was repeatedly shown in the present study, whereas exemplar pictures of a target category were used in the SC IAT.

Procedure. The participants took the three FUMIE Tests during regular psychology classes, in a group, and the IAT with the three target words individually in a psychology laboratory. The order of the two tests was counterbalanced among participants.

\section{Results}

Some participants failed to take the FUMIE Test in the class. The data from 79 ( 31 males and 48 females), 76 (29 males and 47 females), and 77 (28 males and 49 females) participants were used in the following analyses for the targets "marriage," "pregnancy," and "romantic love," respectively.

Treatment of error responses. For the FUMIE Test, we simply counted the completed items without checking their correctness. We randomly sampled $5 \%$ of the participants and determined the error rates for each target. The error rates for the targets "marriage," "pregnancy," and "romantic love" were 0.0037 (4/1,111), $0.0038(4 / 1,061)$, and $0.0(0 / 1,050)$, respectively.

Implicit association scores. The average number of tasks completed in each task and the IASs are listed in Appendix C. The IASs for the three targets were found to be all positive values with statistical significance, showing that the targets were implicitly associated positively among the undergraduate participants $(t \mathrm{~s}=4.84,4.03,6.79 ; p \mathrm{~s}<$ $.001, .01, .001$, respectively). No sex differences were found $(t \mathrm{~s}=0.24,0.62,0.26$; all $p \mathrm{~s}>.10$, respectively).

Validity of the FUMIE Test. The IAT effects were calculated using the D score algorithm (Greenwald et al., 2003), excluding responses under $200 \mathrm{msec}$ and over 5,000 msec from the data analyses. The IASs of the FUMIE Test were compared with the D scores, by participants and for each target, to calculate correlation coefficients. Significant but weak positive correlations between the two test scores were found on all three targets $(r \mathrm{~s}=.35$, $.26, .30 ; N_{\mathrm{s}}=79,76,77 ; \mathrm{ps}<.01, .05, .01$, respectively). Larger correlation coefficients were obtained between the intermediate task data; between the number of items completed in the positive tasks of the FUMIE Test and the mean RT of equivalent tasks of IAT ( $r \mathrm{~s}=-.45,-.61$, -.51 , respectively; all $p \mathrm{~s}<.01$ ), and those of the negative tasks and their equivalents in IAT $(r \mathrm{~s}=-.58,-.61$,
-.64 , respectively; all $p \mathrm{~s}<.01)$. They showed that these tasks had shared the same basic principle. Another correlation study using a prototype version of the bipolar FUMIE Test and an equivalent IAT with 40 undergraduates and 40 senior citizens showed a similar range of correlation coefficients $(r=.32, N=80, p<.01)$ (reported in Mori \& Uchida, 2006). The correlation validity of the FUMIE Test was almost equivalent to that for a paper-format IAT with verbal stimuli $(r \mathrm{~s}=.36-.37, N=70, p<.01)$, and better than that for a paper-format IAT with picture stimuli $(r \mathrm{~s}=$ $.07-.12$, n.s.), both reported by Lemm et al. (2002).

Reliability of the FUMIE Test. Since four identical procedures were repeated in each FUMIE Test, it would be possible to assess the reliability of the test by comparing the results from the first and the last half of the tasks. The correlation coefficient scores (internal consistency correlations) for the three targetwise analyses were $.56, .61$, and .71, for "marriage," "pregnancy," and "romantic love," respectively. These scores were all statistically significant (all $p \mathrm{~s}<.01$ ). Although the correlation coefficients did not reach the level of the conventional criterion of the reliability of psychological tests, they were almost equivalent to the reliability score of IAT (Greenwald et al., 2003), and even higher than the reliability scores calculated for the IAT data in the present study: $.54, .45$, and .60 , respectively.

\section{DISCUSSION}

Although the FUMIE Test showed only weak to moderate correlation coefficients for the validity (between .26 and .35), this does not degrade its validity or reliability, because response latency measures are known to be prone to relatively high measurement error (Cunningham, Preacher, \& Banaji, 2001). It is also worth noting that these positive correlations were better than those obtained from correlation studies assessing other implicit measures, such as the "bona fide pipeline" (Fazio, Jackson, Dunton, \& Williams, 1995) and the IAT. Olson and Fazio (2003) reported that their four studies, with more than 300 participants, altogether revealed little correspondence between the IAT and the BFP ( $r$ s from .5 to -.13 ).

It has been widely known among psychometricians that the reliability of the speed test, to which the IAT and FUMIE Test belong, is moderate in general. It may not be reliable as a psychological test to measure individual traits. However, its results are robust as a group measure. Results from speed tests in general may not be suitable as individual data items, but may well be utilized as group data. In other words, the more data are collected, the more reliable the data become as a whole.

The FUMIE Test is especially suitable for massive data collection. In this respect, it is noteworthy that the FUMIE Test is printed on a single sheet of paper. The test sheet is easily copied or duplicated by a conventional mimeo printer. It is also of crucial importance that it can be administered to a group of participants simultaneously in just $7 \mathrm{~min}$. It requires only a stopwatch for the test administration. This merit of the FUMIE Test may override the intrinsic weak point of the speed tests, their relatively low reliability as psychological tests. Mori and Mori (2007) administered the FUMIE Test 
to all the students of two junior high schools $(N=509)$ to assess implicit attitudes toward three main school subjects: "Japanese," "mathematics," and "English." They found that girls tended to have a less positive attitude toward mathematics than boys. This large data collection was done by asking the schoolteachers to conduct the FUMIE Test in their own classrooms. Its easy administration procedure made this research possible (see Appendix C).

The ease of administration of the FUMIE Test is also a benefit for socially oriented research, in which questionnaires are often used as the main measurement tool. The FUMIE Test can be used in addition to conventional questionnaires for most social research. A combination of explicit and implicit measurements may shed light on unknown aspects of the problem being studied. Imada, Kawamura, and Mori (2006) utilized the FUMIE Test to assess the change in the implicit attitude toward "teacher," "children," and "school" among teachers college students $(N=253)$ before and after class observation. They found unexpected decreases in IASs toward "children" and "school," despite the fact that a conventional questionnaire did not detect any change over time (see Appendix C).

In conclusion, the FUMIE Test can be a viable alternative tool for measuring implicit attitudes. It can be applied to wider research areas and administered more easily than the IAT. As reported above, the FUMIE Test is especially convenient for use in educational settings, such as schools, along with other conventional measures such as questionnaires or term examinations. The FUMIE Test is also useful for preliminary data collection before conducting fullscale investigations with the IAT. Recently, Gawronski, LeBel, and Peters (2007) critically reviewed the validity of the underlying assumption of implicit attitude assessments. The present article would not argue this fundamental problem. However, the new procedure introduced in this article may contribute to examination of the validity of the assumptions of IAT and similar implicit measures, with its merit being the ease of collection of massive amounts of data from various groups of participants in combination with conventional explicit measures.

\section{AUTHOR NOTE}

This research was supported by a grant-in-aid from the Japanese Ministry of Education, Culture, Sports, Science, and Technology (Grant 13610081) while the first author was at Shinshu University, Nagano, Japan. K.M. was greatly encouraged by Anthony G. Greenwald, who expressed interest in his presentation on the FUMIE Test at the 44th Annual Convention of the Japanese Society of Social Psychology, held at Toyo University, Tokyo, in September 2003. We thank all the participants who voluntarily took part in this research. The individual administration of the IAT for validity and reliability assessment was conducted by Yossa Yoshizawa, as part of his graduation thesis submitted to Shinshu University in 2007 , under the supervision of K.M. The data for validity assessment reported in Mori and Uchida (2006) were originally collected by Hisayo Yoneda for her graduation thesis, submitted to Shinshu University in 2004, under the supervision of K.M. We are indebted to Susumu Yamaguchi and Fumio Murakami for their advice in the preparation of the IAT program. We thank Rebecca Ann Marck for her assistance in the preparation of the English manuscript. We also thank the anonymous reviewers of BRM as well as those of Japanese Psychological Research. Their comments were helpful and contributed to the improvement of the article. Correspondence concerning this article should be sent to
K. Mori, Tokyo University of Agriculture and Technology, Koganai, Tokyo, Japan 184-8588 (e-mail: kaz-mori@cc.tuat.ac.jp).

\section{REFERENCES}

Cunningham, W. A., Preacher, K. J., \& Banaji, M. R. (2001). Implicit attitude measures: Consistency, stability, and convergent validity. Psychological Science, 12, 163-170.

FAZio, R. H., JaCKson, J. R., Dunton, B. C., \& Williams, C. J. (1995). Variability in automatic activation as an unobtrusive measure of racial attitudes: A bona fide pipeline? Journal of Personality \& Social Psychology, 69, 1013-1027.

Fazio, R. H., Sanbonmatsu, D. M., Powell, M. C., \& Kardes, F. R. (1986). On the automatic activation of attitudes. Journal of Personality \& Social Psychology, 50, 229-238.

Gawronski, B., LeBel, E. P., \& Peters, K. R. (2007). What do implicit measures tell us? Scrutinizing the validity of three common assumptions. Perspectives on Psychological Science, 2, 181-193.

Greenwald, A. G., McGhee, D. E., \& Schwartz, J. L. K. (1998). Measuring individual differences in implicit cognition: The Implicit Association Test. Journal of Personality \& Social Psychology, 74, 1464-1480.

Greenwald, A. G., NoseK, B. A., \& BanajI, M. R. (2003). Understanding and using the Implicit Association Test: I. An improved scoring algorithm. Journal of Personality \& Social Psychology, 85, 197-216.

Imada, R., KaWAmura, Y., \& Mori, K. (2006, July). Evaluation of implicit attitude toward mono-polar concepts using a paper-and-pencil group performance test. Poster presented at the 4th International Conference on Memory, Sydney, Australia.

KARPINSKI, A., \& SteInMAN, R. B. (2006). The Single Category Implicit Association Test as a measure of implicit social cognition. Journal of Personality \& Social Psychology, 91, 16-32.

Lane, K. A., Mitchell, J. P., \& Banaji, M. R. (2005). Me and my group: Cultural status can disrupt cognitive consistency. Social Cognition, 23, 353-386.

Lemm, K., Sattler, D. N., Khan, S., Mitchell, R. A., \& Dahl, J. (2002, February). Reliability and validity of a paper-based Implicit Association Test. Poster presented at the annual meeting of the Society for Personality and Social Psychology, Savannah, GA.

Lowery, B. S., Hardin, C. D., \& Sinclair, S. (2001). Social influence effects on automatic racial prejudice. Journal of Personality \& Social Psychology, 81, 842-855.

MoRI, K. (2003). The development of the FUMIE Test for measuring the implicit association of target words to negative emotions. Proceedings of the 44th Annual Convention of the Japanese Society for Social Psychology (pp. 104-105).

Mori, K., IMADA, R., \& FuKUnAKA, K. (2005, January). Measuring implicit attitude of mono-polar concepts by a paper-and-pencil test. Poster presented at SARMAC VI (the 6th Conference of the Society for Applied Research in Memory and Cognition), Wellington, New Zealand.

MORI, K., \& Mori, H. (2007). Evaluation of scholastic subjects by junior high school students using a newly developed group performance Implicit Association Test. Annual Letters of Clinical Psychology in Shinshu, 6, 1-4. (in Japanese with an English abstract)

MoRI, K., \& UCHIDA, A. (2006, May). A paper-format group-performance Implicit Association Test. Poster presented at the 18 th Annual Convention of the Association for Psychological Science, New York.

NoseK, B. N., Greenwald, A. G., \& BanaJI, M. R. (2007). The Implicit Association Test at age 7: A methodological and conceptual review. In J. A. Bargh (Ed.), Automatic processes in social thinking and behavior (pp. 265-292). New York: Psychology Press.

Olson, M. A., \& FAZIO, R. H. (2003). Relations between implicit measures of prejudice: What are we measuring? Psychological Science, $14,636-639$.

Schwartz, M. B., O’Neal Chambliss, H., Brownell, K. D., Blair, S. N., \& Billington, C. (2003). Weight bias among health professionals specializing in obesity. Obesity Research, 11, 1033-1039.

Teachman, B. A., Gapinski, K. D., Brownell, K. D., Rawlins, M., \& Jeyarams, S. (2003). Demonstrations of implicit anti-fat bias: The impact of providing causal information and evoking empathy. Health Psychology, 22, 68-78.

Williams, J. M. G., Watts, F. N., MacLeod, C., \& Mathews, A. (1988). Cognitive psychology and social disorders. Chichester, U.K.: Wiley. 


\section{APPENDIX A}

The 16 pairs of words used in the FUMIE Test (the original two Chinese characters, with the English equivalents in parentheses): 平和 (peace)—戦争 (war) 満足 (satisfaction) 一不満 (dissatisfaction) 幸福 (happy)一不幸 (unhappy) 安全 (safety)一危険 (danger) 当選 (elected) 一落選 (eliminated)

勝利 (victory)—敗北 (defeat) 安心 (ease)—不安 (anxiety)

貯金 (savings) 一借金 (debt) 希望 (hope)—絶望 (despair) 長所 (merit) 一短所 (demerit)

平等 (equalization)—差別 (discrimination)

健康 (health) 一病気 (disease)

最高 (best)一最低 (worst)

上品 (superior)—下品 (inferior)

清潔 (clean)—不潔 (dirty)

天使 (angel)一悪魔 (devil)

\section{Supplementary Experiments: Speed Measurements for Marking $\bigcirc$ s and $\times \mathrm{s}$}

EXPERIMENT 1
Simple Speed for Marking Os and $\times \mathbf{s}$
Method
Procedure. Two versions of Task 0, evaluation words without targets, were prepared and administered to 48
undergraduates ( 29 males and 19 females, $20-23$ years old). Half of the participants were asked to mark a circle
on every word within 20 sec on the first task, and then a cross on every word on the second task, within the same
time duration. The other half did the tasks in the reverse order.
Results
The participants marked significantly more circles $(M=45.7, S D=7.40)$ than crosses $(M=39.7, S D=5.16)$
$[t(47)=6.26, p<.001]$. No statistical differences were found for sex, nor for task order. The results showed that
drawing a cross would require about 70 msec more than drawing a circle.

\section{EXPERIMENT 2} Marking Speed in Simulated FUMIE Tasks

Method

Procedure. Two different series of Task 0 were prepared, one with $2 / 3$ positive words and $1 / 3$ negative words, and the other with the reverse proportion. These tasks would require the participants to use similar response patterns as for the positive and negative tasks, respectively. (In the positive tasks, there are $1 / 3$ targets, $1 / 3$ positive words, and 1/3 negative words. Therefore, participants are required to mark a circle on $2 / 3$ of the items.) Forty-eight naive undergraduates (33 males and 15 females, 20-23 years old) took the two series of different tasks twice; first $2 / 3$ positive and $2 / 3$ negative tasks, and then different versions of the same tasks in the reverse order. They were told to mark a circle on a positive word and a cross on a negative word.

\section{Results}

The participants performed similarly on both $2 / 3$ positive and $2 / 3$ negative tasks: $32.1,32.8,36.4$, and 37.3 words, on average, for $2 / 3$ positive, $2 / 3$ negative, $2 / 3$ negative, and $2 / 3$ positive, respectively. No statistical differences were found pairwise, nor in total [all $t \mathrm{~s}(47)<1$, n.s.].

\section{Discussion: Why No Difference in Experiment 2?}

The simple repetition of drawing of circles or crosses may yield a measurable difference in the number of items marked within $20 \mathrm{sec}$ due to accumulation of the small difference, $70 \mathrm{msec}$ or so, in the drawing time for the two marks. However, the small difference will not appear in the actual FUMIE tasks because the task requires not simply drawing marks but making a series of cognitive judgments as well. These judgment times must have absorbed the negligible difference in drawing times. In conclusion, despite the existence of a small drawing-time difference, the marking procedure used in the FUMIE Test would not affect the overall performance speed of the examinees in the practical usage of the test. 
APPENDIX C

Statistics for the Studies Reported

Table C1

Validity and Reliability Assessment Reported in This Article

\begin{tabular}{|c|c|c|c|c|c|c|c|c|}
\hline \multirow[b]{3}{*}{ Target Words } & \multicolumn{6}{|c|}{ Task } & & \\
\hline & \multicolumn{2}{|c|}{ No Targets } & \multicolumn{2}{|c|}{ Positive } & \multicolumn{2}{|c|}{ Negative } & \multicolumn{2}{|c|}{ IAS } \\
\hline & Avg. & $S D$ & Avg. & $S D$ & Avg. & $S D$ & Avg. & $S D$ \\
\hline \multicolumn{9}{|c|}{ Undergraduates $(31,29,28$ Males $)$} \\
\hline Marriage & 35.5 & 6.59 & 35.5 & 6.85 & 30.5 & 5.49 & 4.9 & 3.98 \\
\hline Pregnancy & 35.8 & 5.85 & 34.2 & 7.04 & 32.0 & 5.14 & 2.2 & 5.08 \\
\hline Romantic love & 34.0 & 5.60 & 34.3 & 6.82 & 29.6 & 6.50 & 4.7 & 3.30 \\
\hline \multicolumn{9}{|c|}{ Undergraduates $(48,47,49$ Females $)$} \\
\hline Marriage & 35.0 & 6.72 & 36.7 & 7.17 & 32.2 & 5.68 & 4.4 & 4.22 \\
\hline Pregnancy & 35.1 & 7.81 & 35.7 & 7.25 & 33.6 & 5.13 & 2.1 & 4.77 \\
\hline Romantic love & 35.8 & 7.37 & 34.5 & 7.76 & 30.3 & 5.44 & 4.2 & 4.41 \\
\hline \multicolumn{9}{|c|}{ Undergraduates $(79,76,77$ Total) } \\
\hline Marriage & 35.1 & 8.63 & 36.2 & 7.08 & 31.6 & 6.24 & 4.6 & 4.27 \\
\hline Pregnancy & 35.3 & 6.38 & 35.2 & 6.20 & 33.0 & 6.68 & 2.1 & 5.12 \\
\hline Romantic love & 35.2 & 7.34 & 34.5 & 6.73 & 30.0 & 7.27 & 4.4 & 4.79 \\
\hline
\end{tabular}

Note-Data collected by Yossa Yoshizawa for his graduation thesis submitted to Shinshu University in 2007. The error rates for the targets "marriage," "pregnancy," and "romantic love" were $.0037(4 / 1,111), .0038(4 / 1,061)$, and $0(0 / 1,050)$, respectively.

Table C2

Imada, Kawamura, and Mori (2006)

\begin{tabular}{|c|c|c|c|c|c|c|c|c|}
\hline \multirow{3}{*}{$\begin{array}{l}\text { Target } \\
\text { Words }\end{array}$} & \multicolumn{6}{|c|}{ Task } & & \\
\hline & \multicolumn{2}{|c|}{ No Targets } & \multicolumn{2}{|c|}{ Positive } & \multicolumn{2}{|c|}{ Negative } & \multicolumn{2}{|c|}{ IAS } \\
\hline & Avg. & $S D$ & Avg. & $S D$ & Avg. & $S D$ & Avg. & $S D$ \\
\hline \multicolumn{9}{|c|}{ Freshmen $(N=253)$ : Before-School Visits } \\
\hline Teacher & 41.4 & 7.6 & 33.3 & 6.1 & 29.4 & 4.8 & 3.9 & 4.2 \\
\hline Children & 33.3 & 6.1 & 35.1 & 6.0 & 29.6 & 6.1 & 5.5 & 4.2 \\
\hline School & 34.5 & 6.2 & 35.0 & 6.5 & 29.4 & 5.6 & 5.6 & 4.4 \\
\hline \multicolumn{9}{|c|}{ Freshmen $(N=253)$ : After-School Visits } \\
\hline Teacher & 29.3 & 6.5 & 32.7 & 6.1 & 29.4 & 5.9 & 3.3 & 4.2 \\
\hline Children & 32.2 & 6.4 & 34.8 & 6.1 & 31.1 & 5.4 & 3.7 & 4.6 \\
\hline School & 34.7 & 6.1 & 34.7 & 6.2 & 32.0 & 6.2 & 2.7 & 5.0 \\
\hline
\end{tabular}

Note-The original data were collected in $30 \mathrm{sec}$. The present scores are adjusted for $20 \mathrm{sec}$.

Table C3

Mori (2003)

\begin{tabular}{|c|c|c|c|c|c|c|c|c|}
\hline \multirow[b]{3}{*}{ Target Words } & \multicolumn{6}{|c|}{ Task } & & \\
\hline & \multicolumn{2}{|c|}{ No Targets } & \multicolumn{2}{|c|}{ Incongruent } & \multicolumn{2}{|c|}{ Congruent } & \multicolumn{2}{|c|}{ IAS } \\
\hline & Avg. & $S D$ & Avg. & $S D$ & Avg. & $S D$ & Avg. & $S D$ \\
\hline \multicolumn{9}{|c|}{ Junior High School Students $(N=47)$} \\
\hline Black hair vs. white hair & 25.5 & 5.5 & 24.5 & 5.7 & 24.2 & 5.6 & 0.3 & 3.8 \\
\hline Black people vs. white people & 27.5 & 5.7 & 25.6 & 5.4 & 27.9 & 6.0 & -2.3 & 4.4 \\
\hline Sambo vs. Heidi & 23.9 & 5.3 & 21.7 & 4.9 & 23.7 & 5.5 & -2.0 & 3.2 \\
\hline \multicolumn{9}{|c|}{ Undergraduates $(N=49)$} \\
\hline Black hair vs. white hair & 26.0 & 6.1 & 26.5 & 6.4 & 26.6 & 5.5 & -0.1 & 5.6 \\
\hline Black people vs. white people & 30.8 & 5.4 & 25.0 & 5.4 & 30.2 & 6.5 & -5.2 & 4.7 \\
\hline Sambo vs. Heidi & 26.6 & 6.0 & 25.0 & 5.2 & 26.5 & 5.9 & -1.5 & 3.8 \\
\hline \multicolumn{9}{|c|}{ Adults $(N=52)$} \\
\hline Black hair vs. white hair & 28.7 & 5.2 & 22.5 & 5.4 & 20.5 & 4.0 & 2.0 & 3.6 \\
\hline Black people vs. white people & 30.7 & 4.5 & 22.0 & 4.4 & 28.3 & 6.3 & -6.3 & 4.5 \\
\hline Sambo vs. Heidi & 23.6 & 5.8 & 22.1 & 4.4 & 24.5 & 6.3 & -2.4 & 4.0 \\
\hline
\end{tabular}

Note-A part of the data were collected by Hiromi Ikegami for her graduation thesis submitted to Shinshu University in 2003. The original data were collected in $30 \mathrm{sec}$. The present scores are adjusted for $20 \mathrm{sec}$. 


\section{APPENDIX C (Continued)}

Table C4

Mori, Imada, and Fukunaka (2005)

\begin{tabular}{|c|c|c|c|c|c|c|c|c|}
\hline \multirow{3}{*}{$\begin{array}{l}\text { Target } \\
\text { Words }\end{array}$} & \multicolumn{6}{|c|}{ Task } & & \\
\hline & \multicolumn{2}{|c|}{ No Targets } & \multicolumn{2}{|c|}{ Circle } & \multicolumn{2}{|c|}{ Cross } & \multicolumn{2}{|c|}{ IAS } \\
\hline & Avg. & $S D$ & Avg. & $S D$ & Avg. & $S D$ & Avg. & $S D$ \\
\hline \multicolumn{9}{|c|}{ Undergraduates $(N=34)$} \\
\hline Black & 30.9 & 5.9 & 27.5 & 5.3 & 26.1 & 5.4 & 1.4 & 4.3 \\
\hline
\end{tabular}

$\begin{array}{lllllllll}\text { White } & 30.5 & 6.8 & 29.6 & 5.6 & 26.9 & 6.2 & 2.7 & 3.8\end{array}$

High School Students $(N=33)$

$\begin{array}{lllllllll}\text { Black } & 28.3 & 6.1 & 27.9 & 6.5 & 27.7 & 5.7 & 0.2 & 3.5\end{array}$

$\begin{array}{lllllllll}\text { White } & 28.7 & 5.8 & 28.5 & 6.1 & 25.9 & 5.8 & 2.6 & 4.7\end{array}$

Note-The original data were collected in $30 \mathrm{sec}$. The present scores are adjusted for $20 \mathrm{sec}$.

Table C5

Mori and Mori (2007)

\begin{tabular}{|c|c|c|c|c|c|c|c|c|c|}
\hline \multirow[b]{3}{*}{ Target Words } & \multicolumn{6}{|c|}{ Task } & & & \multirow[b]{3}{*}{ Error Rates } \\
\hline & \multicolumn{2}{|c|}{ No Targets } & \multicolumn{2}{|c|}{ Positive } & \multicolumn{2}{|c|}{ Negative } & \multicolumn{2}{|c|}{ IAS } & \\
\hline & Avg. & $S D$ & Avg. & $S D$ & Avg. & $S D$ & Avg. & $S D$ & \\
\hline \multicolumn{10}{|c|}{ 7th Graders (88 Boys/87 Girls) } \\
\hline Japanese & $25.5 / 26.4$ & $6.53 / 7.09$ & $31.3 / 31.5$ & $6.82 / 6.55$ & $26.3 / 26.6$ & $5.41 / 5.39$ & $5.0 / 4.9$ & $3.96 / 3.88$ & $.004 / .003$ \\
\hline Mathematics & $32.0 / 32.8$ & $5.85 / 5.55$ & $30.1 / 29.5$ & $7.09 / 5.34$ & $27.1 / 27.9$ & $7.17 / 5.34$ & $3.0 / 1.6$ & $5.93 / 5.08$ & $.011 / .008$ \\
\hline English & $34.8 / 34.0$ & $5.64 / 5.30$ & $32.0 / 32.1$ & $6.84 / 6.82$ & $27.5 / 28.0$ & $6.55 / 5.80$ & $4.5 / 4.1$ & $3.82 / 4.30$ & $.009 / .006$ \\
\hline \multicolumn{10}{|c|}{ 8th Graders (80 Boys/84 Girls) } \\
\hline Japanese & $26.1 / 27.1$ & $6.79 / 6.32$ & $33.0 / 33.3$ & $7.18 / 7.27$ & $27.1 / 27.6$ & $5.61 / 6.48$ & $5.9 / 5.7$ & $4.04 / 4.22$ & $.007 / .028$ \\
\hline Mathematics & $32.8 / 33.6$ & $7.01 / 5.81$ & $31.9 / 32.0$ & $7.25 / 7.50$ & $27.5 / 28.4$ & $5.95 / 6.13$ & $4.4 / 3.6$ & $4.74 / 4.67$ & $.009 / .013$ \\
\hline English & $35.4 / 35.4$ & $7.37 / 6.97$ & $34.0 / 34.1$ & $7.74 / 6.86$ & $29.1 / 29.1$ & $5.81 / 5.44$ & $4.9 / 5.0$ & $4.45 / 4.11$ & $.014 / .014$ \\
\hline \multicolumn{10}{|c|}{ 9th Graders (89 Boys/81 Girls) } \\
\hline Japanese & $29.7 / 28.7$ & $8.40 / 7.63$ & $35.2 / 35.0$ & $7.08 / 7.08$ & $28.9 / 29.4$ & $6.25 / 6.14$ & $6.3 / 5.6$ & $4.24 / 4.67$ & $.012 / .006$ \\
\hline Mathematics & $35.6 / 35.2$ & $6.09 / 6.38$ & $34.8 / 33.4$ & $7.82 / 6.90$ & $29.3 / 31.2$ & $6.60 / 5.58$ & $5.5 / 2.2$ & $5.14 / 4.72$ & $.007 / .019$ \\
\hline English & $36.9 / 36.8$ & $7.55 / 6.34$ & $36.8 / 36.7$ & $6.78 / 7.03$ & $31.5 / 31.0$ & $6.20 / 6.20$ & $5.3 / 5.7$ & $4.90 / 5.79$ & $.007 / .006$ \\
\hline
\end{tabular}

Table C6

Mori and Uchida (2006)

\begin{tabular}{|c|c|c|c|c|c|c|c|c|c|}
\hline \multirow{3}{*}{$\begin{array}{l}\text { Target } \\
\text { Words }\end{array}$} & \multicolumn{6}{|c|}{ Task } & \multirow{2}{*}{\multicolumn{2}{|c|}{ IAS }} & \multirow[b]{3}{*}{ Error Rates } \\
\hline & \multicolumn{2}{|c|}{ No Targets } & \multicolumn{2}{|c|}{ Incongruent } & \multicolumn{2}{|c|}{ Congruent } & & & \\
\hline & Avg. & $S D$ & Avg. & $S D$ & Avg. & $S D$ & Avg. & $S D$ & \\
\hline \multicolumn{10}{|c|}{ Senior Citizens (20 Males/20 Females) } \\
\hline Black people vs. white people & $17.5 / 16.3$ & $6.4 / 6.3$ & $14.9 / 13.8$ & $6.0 / 6.9$ & $15.4 / 15.2$ & $5.8 / 6.2$ & $-0.5 /-1.4$ & $3.8 / 4.5$ & $.0331 / .0393$ \\
\hline \multicolumn{10}{|c|}{ Undergraduates (20 Males/20 Females) } \\
\hline Black people vs. white people & $28.3 / 27.9$ & $5.1 / 5.5$ & $25.4 / 26.3$ & $6.1 / 5.8$ & $25.9 / 25.5$ & $5.7 / 6.1$ & $-0.5 / 0.8$ & $3.9 / 3.1$ & $.0189 / .0178$ \\
\hline
\end{tabular}

Note-Data collected by Hisayo Yoneda for her graduation thesis submitted to Shinshu University in 2004 . The original data were collected in 30 sec. The present scores are adjusted for $20 \mathrm{sec}$.

(Manuscript received October 15, 2006;

revision accepted for publication November 23, 2007.) 